\title{
Dynamics of Viscous Amphiphilic Films Supported by Elastic Solid Substrates.
}

\author{
M. V. Voinova ${ }^{1,2}$, M. Jonson ${ }^{2}$ and B. Kasemo ${ }^{2}$ \\ ${ }^{1}$ Department of Theoretical Physics, Kharkov State University, \\ Kharkov 310077, Ukraine \\ 2 Department of Applied Physics, Chalmers University of Technology and Göteborg University, \\ S-412 96, Göteborg, Sweden
}

(Revised version: 6 June 1997)

\begin{abstract}
The dynamics of amphiphilic films deposited on a solid surface is analyzed for the case when shear oscillations of the solid surface are excited. The two cases of surface- and bulk shear waves are studied with the film exposed to gas or to a liquid. By solving the corresponding dispersion equation and the wave equation while maintaining the energy balance we are able to connect the surface density and the shear viscosity of a fluid amphiphilic overlayer with experimentally accessible damping coefficient, phase velocity, dissipation factor and resonant frequency shifts of shear waves.
\end{abstract}

PACS number(s): 68.15.+p, 68.60.Bs, 43.35.Bf 


\section{INTRODUCTION.}

Measurements of the properties of thin adsorbed films have since long been an important subfield of interface studies [1 10]. Scientific and technological applications of thin films like Langmuir-Blodgett (LB) films [11 20], self-assembled monolayers (SAM) 21] and protein monolayers and multilayers [22,23] have stimulated detailed studies of the physical properties of such structures. With these model systems it is possible to construct multilayer amphiphilic films with controlled monolayer thickness $h_{j} \simeq 25 \AA$ and to create different coupling situations between the adsorbed film and the chemically modified substrate [12 [16]. Specifically measurements of surface density and viscosity of these films are important both scientifically and, e.g., with respect to their possible application in acoustical biosensors in which they can serve as sensitive elements [13, 16, 18, 19.

The dynamic (shear) viscosity of amphiphilic films is a very important rheological characteristic of a protein film or a fluid membrane, which is strongly dependent on temperature and phase state of the film, and on environmental conditions [13,24,25]. Usually LB or SAM film acoustic sensors operate in vacuum or in a gaseous enviroment. In contrast, a lipid-water system or a protein layer adsorbed onto a solid surface in a fluid experiences "wet" conditions or a bulk aqueous medium $[23,26]$ at room temperature, which can change the viscosity of the film. In addition, the biomolecular layer adsorption process is extremely sensitive to the nature of the solid substrate in the sense that it can modify the overlayer structure [13,16,20 22]. In view of this, a rigorous description of the dynamics of the existing interfaces of combined solid substrate-adsorbed layer-bulk liquid systems, as well as of solid surface-amphiphilic film-gas systems seems to be greatly needed.

In the present paper we investigate the dynamics of thin amphiphilic layer(s) attached to a solid substrate oscillating in a shear mode and in contact with (i) a gas or (ii) a bulk newtonian liquid. Figs. (1-3) show schematically the geometry of such sandwich systems.

Amphiphilic bilayer films can imitate the behavior of a bilayer lipid membrane. The constituent bilayer molecules are composed of hydrophilic groups attached to hydropho- 
bic chains of different length. The phase diagrams of such amphiphilic molecules demonstrate a rich variety of properties and behavior depending on temperature and water content $[13,20,24,27,28]$. Above the liquid crystalline-to-gel transition the hydrocarbon chains are approximately liquid and a bilayer membrane behaves like a two-dimensional fluid in the lateral plane due to the vanishing shear modulus of elasticity [27,28]. Such a peculiar fluid is isotropic in its plane but it is anisotropic in the normal direction due to sublayered headand-tail structure [24]. Viscous layer response on shear deformation can be characterized by different shear viscosity coefficients, namely, the surface viscosity, $\eta_{s}[13]$, and a bulk shear viscosity, $\eta_{M}$ (a component of tensor $\eta_{j k l m}$, index 'M' corresponds to the classification according to Ref. 28).

When the system is immersed in water, anisotropy can arise from water being trapped in the layer. This can result in a value of the overlayer viscosity $\eta_{M}$, which is distinctly different compared to the case of a gaseous (air) enviroment. This is especially important for protein adsorbed layers with nonuniform interfacial domain structure and nonuniform water distribution in the normal direction. Such nonuniformity effects have been analyzed in a recently published hydrodynamic model of "porous" polymer surface film in liquids (for a review see Ref. 26 and references therein).

In the present paper we have considered both the surface viscosity $\eta_{s}$ and the bulk shear viscosity $\eta_{M}$ of adsorbed amphiphilic films. These can be measured in different acoustic experiment, when acoustic shear waves propagate along the plane of the layer (horizontally polarized surface shear waves) and in the direction normal to it (bulk acoustic shear waves). In order to be able to describe both air- and liquid enviromental experiments, corresponding shear viscosity coefficients, $\eta_{M}{ }^{\prime}$ in air' and $\eta_{M}{ }^{\prime}$ in liquid' have been noted.

The paper is organized as follows: in the framework of continuum mechanics we derive in Section II the dispersion equation for elastic shear waves propagating in the system. We also treat the interaction between horizontally polarized elastic surface shear waves (SH SSW) and a fluid layer adsorbed on the surface of a semi-infinite solid substrate. In Section III 
we analyze the response of bulk acoustic shear waves (BAW) propagating in a finite quartz plate with a viscous overlayer. Our results allow us to connect the surface density and shear viscosity of amphiphilic overlayers with experimentally accessible damping coefficient, phase velocity, dissipation factor and resonant frequency shift of shear waves. These can be measured by modern piezo-acoustical devices of different types [23, 26, 29].

\section{DYNAMICS OF A VISCOUS BILAYER FILM ON A SOLID SUBSTRATE OSCILLATING IN A GASEOUS MEDIUM OR IN VACUUM.}

Within continuum mechanics [30,31] the dynamics of a liquid film can be described by the equation

$$
\rho \frac{d v_{j}}{d t}=\rho\left(\partial v_{j} / \partial t+(\mathbf{v} \nabla) v_{j}\right)=\partial_{k} \sigma_{j k}
$$

This corresponds to the Navier-Stokes equation for the motion of a viscous liquid. Recently it was shown that hydrodynamical properties of fluid amphiphilic (lipid) films can be analyzed within the continuum mechanics scheme with viscous tensions defined as follows [28]:

$$
\begin{gathered}
\sigma_{j k}=\mathcal{A}_{l m} \eta_{j k l m} \\
\mathcal{A}_{l m}=\frac{1}{2}\left(\partial_{l} v_{m}+\partial_{m} v_{l}\right), \partial_{l} \equiv \partial / \partial x_{l} .
\end{gathered}
$$

Here $\eta_{j k l m}$ is a viscosity matrix which were introduced explicitly in Ref. [32].

For a fluid lipid layer in the incompressible liquid approximation and in the absence of pressure gradient, these expressions can be simplified [28]. In particular, for the case when a strain is applying along the $x$ direction, one finds

$$
\sigma_{x y}=\eta_{M}\left(\partial_{y} v_{x}\right)
$$

Here the viscosity coefficient $\eta_{M}$ is a component of the viscosity matrix $\eta_{j k l m}$; the $y$-axis is perpendicular to the layer plane.

In our model we use a notation $\eta_{M}$ (liquid) and $\eta_{M}$ (gas) for describing fluid films bounded (in different experiments) by a liquid or a gas. 
Below we analyze the influence of viscosity and surface density of a thin bilayer on the phase velocity and the damping of elastic shear waves.

\section{Surface Shear Wave Propagation: The Semi-infinite Quartz Crystal Substrate}

We treat here horizontally polarized surface elastic waves on a solid substrate interacting with a thin fluid double layer, which on the other side has an interface to vacuum or to a gaseous phase (Fig.1). Surface waves on plane interfaces have an amplitude which decays exponentially with normal distance from the solid surface on which they propagate [2]. Phase velocities of surface shear waves (SSW) $V$ are lower than those of bulk shear waves $V_{0}$ in the semi-infinite elastic half-space (i.e. the substrate).

Let us now consider how the shear vibration of the substrate generates a viscous wave in the adjacent fluid layer [2,0,17]. In the framework of fluid mechanics, the motion of an adsorbed viscous film is described by the linearized Navier-Stokes equation:

$$
\begin{aligned}
& \left.\partial v_{x}(y, z, t)\right) / \partial t=\nu_{1} \Delta v_{x}, \\
& \nu_{1} \equiv \eta_{1} / \rho_{1}, \\
& \eta_{1}=\eta_{M}(\text { gas }) .
\end{aligned}
$$

Equation (1) is valid in the regime of small Reynolds numbers.

$$
R=\frac{\omega u_{0} h_{1}}{\nu_{1}} \ll 1
$$

for small oscillation frequency $\omega$ and -amplitude $u_{0}$ and for a thin overlayer thickness $h_{1}$. Here $v_{x}$ denotes the $x$-component of the velocity of the fluid film and the $y$-axis is perpendicular to the $z$-direction of wave propagation.

The boundary conditions at the fluid-solid interface $(y=0)$ correspond to the assumption of no slip conditions $[6,26,28,30]$

$$
\begin{array}{r}
y=0: \quad v_{x}=\frac{\partial u_{x}(y, z, t)}{\partial t}, \\
\sigma_{y x}=\eta_{1} \frac{\partial v_{x}}{\partial y}, \\
\sigma_{y x}=C_{44} \frac{\partial u_{x}}{\partial y} .
\end{array}
$$


Here $u_{x}$ is a component of the substrate boundary displacement vector,

$$
u_{x}=u_{0} \exp \left(\kappa_{t} y+i q z\right) \exp (i \omega t)
$$

and $\kappa_{t}=\left(q^{2}-\rho_{0} \omega^{2} / C_{44}\right)^{1 / 2}$ is the inverse penetration depth of surface shear waves into the solid substrate; $\omega, q$ are wave frequency and wave number, while $\rho_{0}$ and $C_{44}$ are the substrate density and shear modulus, respectively. The substrate boundary motion is described by the equation

$$
\rho_{0} \ddot{u_{x}}=C_{44}\left(\frac{\partial^{2} u_{x}}{\partial y^{2}}+\frac{\partial^{2} u_{x}}{\partial z^{2}}\right)
$$

which corresponds to elastic vibrations of the solid surface.

Boundary conditions at the moving interface between fluid 1 and fluid $2\left(y=h_{1}\right.$, Fig. 1) follow from the condition that the friction forces must be the same [30]:

$$
\begin{array}{r}
y=h_{1}, \quad n_{k} \sigma_{i k}^{(1)}=n_{k} \sigma_{i k}^{(2)}, \\
\sigma_{i k}^{(1)}=-p_{1} \delta_{i k}+\eta_{1}\left(\frac{\partial v_{i}^{(1)}}{\partial x_{k}}+\frac{\partial v_{k}(1)}{\partial x_{i}}\right), \\
\sigma_{i k}^{(2)}=-p_{2} \delta_{i k}+\eta_{2}\left(\frac{\partial v_{i}^{(2)}}{\partial x_{k}}+\frac{\partial v_{k}(2)}{\partial x_{i}}\right), \\
v_{x}^{(1)}=v_{x}^{(2)}, \quad v_{z}^{(1)}=v_{z}^{(2)}=0,
\end{array}
$$

At the free surface, finally, the boundary condition is:

$$
y=h_{2}, \quad \eta_{2} \frac{\partial v_{x}^{(2)}}{\partial y}=0
$$

Equations (1-4) together with the equation for the elastic substrate motion lead to the following dispersion equation for horizontally polarized surface shear waves in our system:

$$
\kappa_{t}=\frac{i \omega \eta_{1} \xi_{1}}{C_{44}} \frac{\left(\frac{\xi_{1}}{\xi_{2}}+\epsilon \tanh \left(\Delta h \xi_{2}\right)\right) \exp \left(2 h \xi_{1}\right)-\left(\frac{\xi_{1}}{\xi_{2}}-\epsilon \tanh \left(\Delta h \xi_{2}\right)\right)}{\left(\frac{\xi_{1}}{\xi_{2}}+\epsilon \tanh \left(\Delta h \xi_{2}\right)\right) \exp \left(2 h \xi_{1}\right)+\left(\frac{\xi_{1}}{\xi_{2}}-\epsilon \tanh \left(\Delta h \xi_{2}\right)\right)}
$$

where $\xi_{j}=\left(q^{2}+i \omega / \nu_{j}\right)^{1 / 2}, \quad j=1,2, \epsilon \equiv \eta_{2} / \eta_{1}$, and $\Delta h=h_{2}-h_{1}$.

Here we introduce the viscous penetration depth $\delta \equiv(2 \nu / \omega)^{1 / 2}$ corresponding to the distance over which the transverse wave amplitude falls off by factor of $e$. For two sufficiently thin viscous overlayers one can assume that $h_{j} / \delta_{j} \ll 1$, and we will always consider the long wavelength limit, where $q^{2} \ll \delta^{-2}$. Within these limits, we can from Eqn. (5) find the SSW damping coefficient as a function of the viscosities and the surface densities of the overlayers. 
The result for the damping coefficient $\Gamma$ of the SSW — simply the imaginary part of the wave vector $q-$ is

$$
\Gamma=\Gamma_{m o n}\left\{1+\frac{\eta_{2 s}}{\eta_{1 s}}\left(1+\frac{\eta_{1 s} \rho_{2 s}}{\eta_{2 s} \rho_{1 s}}\left(1+\frac{\eta_{2 s}}{\eta_{1 s}}\right)\right)\right\} .
$$

Here $\eta_{j s} \equiv \eta_{M} h_{j}$ is the surface viscosity component of each layer, $\rho_{j s} \equiv \rho_{j} h_{j}$ is a corresponding surface density, and $\Gamma_{\text {mon }}$ denotes the damping coefficient for the case of a monolayer [17,

$$
\Gamma_{m o n}=\frac{q_{0} \omega^{3} \rho_{1 s} \eta_{1 s}}{C_{44}^{2}}
$$

One can estimate the value of $\Gamma$ to be $\sim 10^{-5}$ for a phospholipid bilayer. Experimental data for egg lecithin at $T=25^{\circ} \mathrm{C}$ are taken from Ref. [24], which provides the following values: bilayer thickness $h_{b}=46 \AA$, bilayer density $\rho \approx 1 \mathrm{~g} / \mathrm{cm}^{3}$, bilayer "microviscosity" (measured by a probe technique) $\eta \approx 1.2 \mathrm{dyn} \cdot \mathrm{s} / \mathrm{cm}^{2}$. The resonator frequency is $\omega_{0} \approx 10^{9} \mathrm{~Hz}$ and $C_{44} \approx 2.6 \mathrm{dyn} / \mathrm{cm}^{2}$ is the corresponding quartz shear modulus.

Using the dispersion equation (5) one can also obtain the change in the SSW phase velocity, $\Delta V / V_{0}$, caused by the presence of the adsorbed bilayer. One finds that

$$
\begin{gathered}
\frac{\Delta V}{V_{0}} \approx \frac{1}{2}\left(\frac{\omega \rho_{s_{1}}}{V_{0} \rho_{0}}\right)^{2}\left(1+\frac{\rho_{s_{2}}}{\rho_{s_{1}}}\right)^{2} \\
V_{0} \equiv \sqrt{C_{44} / \rho_{0}}=\text { const. }
\end{gathered}
$$

As a concequence of the no slip- and thin layer assumptions, the SSW velocity shift is sensitive only to the overlayer surface densities $\rho_{s_{j}}$ and not to their viscosities. This corresponds to the Love type of wave propagation. Equation (7) allows one to determine the surface density of the upper or lower half of the bilayer if the density of the other layer is known or can be determined by an independent experiment. For the lipid bilayer (with the same parameters as above) the SSW phase velocity shift is small, $\Delta V / V_{0} \sim 10^{-8}$. However, this shift may be detectable (of order $\sim 10^{-6}$ ) at high frequences, $\omega \approx 2 \pi 10^{9} \mathrm{~Hz}$, since the SSW velocity change is a quadratic function of frequency. Such high frequencies can be

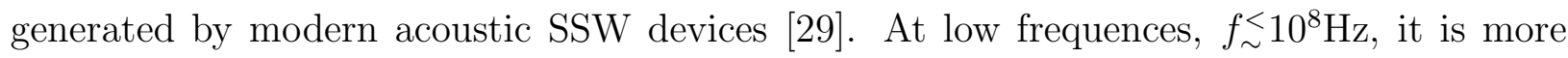


reasonable to use another type of shear waves for surface density analysis excited by quartz crystals oscillating in the thickness shear mode (see Section III.B below).

\section{SOLID SUBSTRATE WITH VISCOUS OVERLAYER OSCILLATING IN A BULK VISCOUS MEDIUM.}

\section{Resonant Frequency and Dissipation Factor for Bulk Shear Waves: The Finite Quartz-Crystal Resonator}

In addition to "genuine" surface waves, another type of shear waves can propagate in the plane of the overlayer-substrate interface. These are bulk acoustic waves (BAW). In contrast to the SSW case considered above, the acoustical response of unloaded BAW resonators depends on the quartz slab thickness $h_{0}$ as well as on its density $\rho_{0}$ and shear modulus $C_{66}$

$$
f_{0}=\left(C_{66} / \rho_{0}\right)^{1 / 2} h_{0}
$$

It has been shown [3, 6, 23, 26, 33 that the resonant frequency of such quartz plates decreases when its surface is coated with an overlayer constituting a mass load. For a thin surface film, uniformly covering the entire vibrating area of the slab, Sauerbrey deduced a linear relation between the frequency change and the added mass per unit area in a vacuum (gaseous) environment [3]. Kanazawa and Gordon [6] have later described the resonant response of quartz oscillators in bulk viscous liquids. Recent experimental and theoretical investigations of the BAW in composite media (see Refs. 23, 26, 33 for a review) have demonstrated possible applications to the study of nonuniform polymer and protein thin films and have included both resonant frequency and viscous dissipation in the interface region. Experimentally, it is convenient to obtain this viscous dissipation as the width of the resonant frequency [26] or as the dissipation factor $D[23]$

$$
D=E_{\text {dissipated }} / 2 \pi E_{\text {stored }}=1 / \pi f \tau
$$

where $\tau$ is the time constant for the decay of the vibration amplitude. 
In this Section we calculate the shifts $\Delta f$ of the resonance frequency and $\Delta D$ of the dissipation factor, respectively, for the case of a viscous amphiphilic (lipid) film attached without slip to the surface of the quartz slab oscillating in the thickness shear mode in the presence of a bulk viscous liquid.

The wave equation for shear waves propagating in the vertical direction (Fig.2) is

$$
\frac{\partial^{2} u_{x}(y, t)}{\partial y^{2}}=\frac{i \rho_{1} \omega}{\eta_{1}} u_{x}(y, t), \quad \eta_{1} \equiv \eta_{M}(\text { liquid })
$$

Its general solution has the form

$$
\begin{gathered}
u_{x}=e^{i \omega t}\left(U_{1} e^{-\xi_{1} y}+U_{2} e^{\xi_{1} y}\right) \\
\xi_{1}=(1+i) / \delta_{1}
\end{gathered}
$$

Using the same boundary conditions (2-4) as above, we find for the $x$-component of the liquid velocity:

$$
\begin{gathered}
v_{x}=v_{0} \frac{e^{\xi_{1} y}+A e^{-\xi_{1}\left(y-2 h_{1}\right)}}{1+A e^{2 h_{1} \xi_{1}}} \\
A=\frac{\delta_{2}+\epsilon \delta_{1} \tanh \left[(1+i) \Delta h / \delta_{2}\right]}{\delta_{2}-\epsilon \delta_{1} \tanh \left[(1+i) \Delta h / \delta_{2}\right]}
\end{gathered}
$$

The shift of resonant frequency $\Delta f$ and dissipation factor $\Delta D$, due to the overlayer on the substrate, can be calculated from the balance between dissipated and stored energy in the system 33,26,23. Using this energy balance, we find from Eq. (11):

$$
\begin{aligned}
& \Delta f \approx-I m\left(\eta_{1} \xi_{1} \frac{A e^{2 h_{1} \xi_{1}}-1}{A e^{2 h_{1} \xi_{1}}+1}\right) / 2 \pi \rho_{0} h_{0} \\
& \Delta D \approx-\operatorname{Re}\left(\eta_{1} \xi_{1} \frac{A e^{2 h_{1} \xi_{1}}-1}{A e^{2 h_{1} \xi_{1}}+1}\right) / \pi f \rho_{0} h_{0}
\end{aligned}
$$

In the limiting case $h_{1} / \delta_{1} \ll 1, \Delta h / \delta_{2} \gg 1$ we obtain for the resonant frequency shift

$$
\Delta f_{\text {res }}=-f_{0}\left(\frac{f \eta_{2} \rho_{2}}{\pi \rho_{0} C_{66}}\right)^{1 / 2}\left\{1+h_{1} \rho_{1}\left(\frac{4 \pi f}{\eta_{2} \rho_{2}}\right)^{1 / 2}-\left(4 \pi f \eta_{2} \rho_{2}\right)^{1 / 2} \frac{h_{1}}{\eta_{1}}\right\}
$$


This expression contains contributions from both the lower and the upper layer. For sufficiently viscous solutions, $\eta_{2} \approx \eta_{1} \rho_{1} / \rho_{2}$, the second and third terms may be of the same order.

In contrast, the result for $\Delta D$ is practically insensitive to the ultrathin overlayer parameters. In the linear approximation, corresponding to a small value of $h_{1} / \delta_{1}$, it depends only on the upper (bulk) liquid viscosity and its density:

$$
\Delta D=2 f_{0}\left(\frac{\eta_{2} \rho_{2}}{\pi f \rho_{0} C_{66}}\right)^{1 / 2}\left\{1-\frac{2 \pi h_{1}^{2} \rho_{1} f}{\eta_{1}}\left(\frac{\eta_{2} \rho_{2}}{\eta_{1} \rho_{1}}-1\right)\right\}
$$

In the limit of $\eta_{2} \rightarrow 0$ we obtain the Sauerbrey formula:

$$
\Delta f=-\frac{2 f_{0}^{2} \rho h}{\sqrt{\rho_{0} C_{66}}} .
$$

In the opposite case and for $h_{1}=0$, the results of Kanazawa and Gordon follow:

$$
\Delta f_{\text {res }}=-f_{0}\left(\frac{f \eta_{2} \rho_{2}}{\pi \rho_{0} C_{66}}\right)^{1 / 2} .
$$

Formulae (14) and (15) form a set of equations. From these expressions it is possible to determine the $\eta_{M}$ component of the overlayer shear viscosity and the thickness (mass) of the adsorbed layer and compare them with the results of surface viscosity measurements. Also, it is possible to determine the viscosity and density of the adjacent bulk liquid if parameters of the overlayer are known.

It is essential to note that our model is valid for an arbitrary ratio $\epsilon$ between the viscosities $\eta_{2}$ and $\eta_{1}$, i.e., even for $\epsilon \geq 1$. The latter possibility may be relevant for a thin amphiphilic substrate-adjacent film under a thick protein layer (For example, a sandwich structure such as the one schematically shown in Fig.4. can be realised by an adsorbed protein layer attached to the substrate via an amphiphilic self-assembling monolayer [22]).

\section{DISCUSSION.}

In addition to its importance in technological applications, the study of the hydrodynamic modes of fluid amphiphilic films and adsorbed proteins are generally important for 
understanding the dynamics of the swelling of lipid-water systems $[28,34,35]$ and the rheology of biological membranes. It is known, for instance, that living cell membranes, e.g. in red blood cells, experience shear stress in hydrodynamic flow through capillaries [36, 37]. As the lipid bilayer matrix of the membrane is a two-dimensional incompressible liquid adjacent to an elastic protein (spectrin-actin) network, the hydrodynamics of this layered structure is governed by the coupling of the fluid membrane to shear flows in an external bulk liquid 36,37 .

The surface viscosity of a fluid amphiphilic film is often considered to be a twodimensional analogue of the bulk viscosity and defined as the coefficient of proportionality between the tangential force per unit length and the gradient in the flow velocity of the liquid. These averaged surface characteristics can be measured, e.g. by an interface shear rheometer at air-liquid interfaces [13,25] or by using the oscillating barrier method in a LB trough [25]. In contrast to $\eta_{s}$, the "microscopic" shear viscosity may be defined as a contribution of fluid disordered chains. The "microviscosity" of the membrane core can be determined experimentally, e.g. by a probe technique, and describes the local viscosity near the probe [24]. This "microviscosity" is often identified with the true membrane viscosity. However, this experimental technique could give rather different results with a strong dependence on the probe material and on the interaction between the probe and its local surroundings [24]. Both of these viscosities may be changed after the deposition of an amphiphilic layer on the solid surface.

The results of our theory can be applied for direct acoustic measurements of surface densities and/or shear viscosities $\eta_{s}$ and $\eta_{M}$ for a thin amphiphilic film after the transferring onto the solid substrate and in both the liquid and gaseous experimental conditions. Another help is a possible strong coupling between the end groups of lipid layer, LB film or SAM and the surface of the substrate [12-16], thus for the interfacial solid-fluid region, no slip assumption seems to be valid.

In our work we found the dispersion equation for surface shear acoustic waves with 
horizontal polarization and solved it as well as the wave equation for shear bulk acoustic waves. The solution of these equations together with energy balance, allows us to calculate the analytical expressions for damping coefficients, phase velocities, dissipation factor and resonant frequency shifts of both types of shear waves as functions of lipid film surface density and two different components of its in-plane viscosity. These acoustic waves can be excited by means of two different types of piezoelectric oscillators [23,29].

We can suggest to measure the acoustical responce of the above-mentioned sandwich system in the region of lipid phase transition when the viscosity of the overlayer subject to dramatic changes. For instance, during the liquid crystalline to gel transformation of a lipid membrane, the viscosity of the bilayer changes by more than 10 times while the membrane density remains practically constant [17,24]. It is an interesting experimental fact [34] that the two halves of a bilayer lipid membrane are so weakly coupled, that they can udergo the thermotropic phase transition independently. In the accordance with our results, it must provide the changes in SSW damping coefficient, while the corresponding SSW phase velocity shift due to the presence of bilayer will be constant.

On a more speculative note, our results may also be valid within the segregated (sublayer) structure model of adsorbed protein layers (Fig. 3). In accordance with this model (see, e.g. $[21,22])$, the structure of the protein overlayer resembles a surfactant film composed of segregated head-and-tail regions. Recent neutron scattering experiments 22] have given evidence for such an anisotropic sublayer structure of protein monolayers adsorbed onto solid surfaces through the self-assembling amphiphilic sublayer. In this case, the relation between the upper and lower layer viscosities may vary in strong dependence of the phase state of protein and amphiphilic monolayer, respectively. 


\section{ACKNOWLEDGEMENTS.}

One of us (MV) is grateful to Prof. S .F. Edwards for the possibility of visiting the Polymer and Colloid Group at the Cavendish Laboratory. We gratefully acknowledge detailed discussions about quartz crystal microbalance experiments with M. Rodahl and A. Krozer $(\mathrm{CTH} / \mathrm{GU})$ and helpful correspondence concerning recent neutron scattering data on adsorbed proteins with A. R. Rennie (Cambridge).

This work was supported by the Royal Swedish Academy of Sciences (KVA). 


\section{REFERENCES}

[1] Fluid Interfacial Phenomena 1986 ed C A Croxton (Chichester, UK : J.Wiley and Sons)

[2] Uberall H 1988 Physical Acoustics: Principles and methods ed W P Mason and R N Thurston (New York: Academic P.) 18

[3] Sauerbrey G 1959 Z.Phys. 155206

[4] Earnshaw J C and Winch P J 1990 J.Phys.:Condens.Matter 28499

[5] Kosevich Yu A and Syrkin E S 1990 J.Phys.:Condens.Matter 25047

[6] Kanazawa K K and Gordon J G 1985 Anal.Chem. 571770

[7] Mitchell D J, Ninham B W and Pallthorpe B A 1978 J.Colloid Interface Sci. 64194

[8] Reed C E , Kanazawa K K and Kaufman J H 1990 J.Appl.Phys. 681993

[9] Duncan-Hewitt W C and Thompson M 1992 Analyt.Chem. 6494

[10] Krim J and Widom A 1988 Phys.Rev.B 3812184.

[11] Johannsmann D, Mathauer K, Wegner G and Knoll W 1992 Phys.Rev. B 467808

[12] Bordieu L, Silberzan P and Chaternay D. 1991 Phys.Rev.Lett. 672029

[13] Blinov L M 1988 Sov.Phys.-Uspekhi 155443

[14] Bareman J P, Cardini G and Klein M L 1988 Phys.Rev.Lett. 602152

[15] Decher G, Maclennan J, Sohling U and Reibel J 1992 Thin Solid Films 210/211 504

[16] Voinova M V , Kosevich A M and Syrkin E S 1993 Sov.Phys.-Acoust. 39944

[17] Voinova M V and Syrkin E S 1996 Sov. Phys.-Crystallography in press

[18] Ohnishi M, Ishimoto C and Seto J 1992 Thin Solid Films 210/211 455

[19] Kosevich A M, Syrkin E S and Voinova M V 1996 Acoustical Imaging ed J P Jones 
(New York:Plenum Publ.) 21210

[20] Vogel V and Woll C 1986 J.Chem.Phys. 845200

Bechhoefer J, Jerome B and Pieranski P 1991 Phase Transit. B 33227

[21] McDermott D C, Kanelleas D, Thomas R K, Rennie A R, Satija S K and Majkrzak C F 1993 Langmuir 92404

[22] Fragneto G, Thomas R K, Rennie A R and Penfold J 1995 Science 267657

[23] Rodahl M, Hook F, Krozer A, Brzezinski P and Kasemo B 1995 Rev.Sci.Instr. 663924

[24] Ivkov V G and Berestovski G N 1981 Dynamical structure of lipid bilayer (Moscow: Nauka)

[25] Kragel J, Li J B, Miller R, Bree M, Kretzschmar G and Mohwald H 1996 Colloid Polym Sci 2741183

[26] Daikhin L and Urbakh M 1996 Langmuir 126354

[27] Helfrich W 1974 Phys Lett A50 115

[28] de Gennes P - G 1992 Simple Views on Condensed Matter (Singapore: World Sci.Publ.Co.Pte.Ltd.)

[29] Plesski V P and Ten Yu A 1984 Sov.Phys.-Tech.Phys.Lett. 10124

[30] Landau L D and Lifshitz E M 1959 Fluid Mechanics (Pergamon Press)

[31] Landau L D and Lifshitz E M 1987 Theory of Elastisity (Moskow: Nauka)

[32] Martin P C, Parodi O and Pershan P S 1972 Phys.Rev.A 62401

[33] Mecea V M 1993 Sensors and Actuators A40 1

[34] Sillerud L O and Barnett R E 1982 Biochemistry 211756

[35] Noirez L and Lapp A 1996 PRL 7870 
[36] Evans E A and Skalak R 1980 Mechanics and Thermodynamics of Membranes (Florida: CRC Press, Inc.,Boca Raton)

[37] Kraus M, Wintz W, Seifert V and Lipowsky R 1996 PRL 773685 


\section{FIGURES}

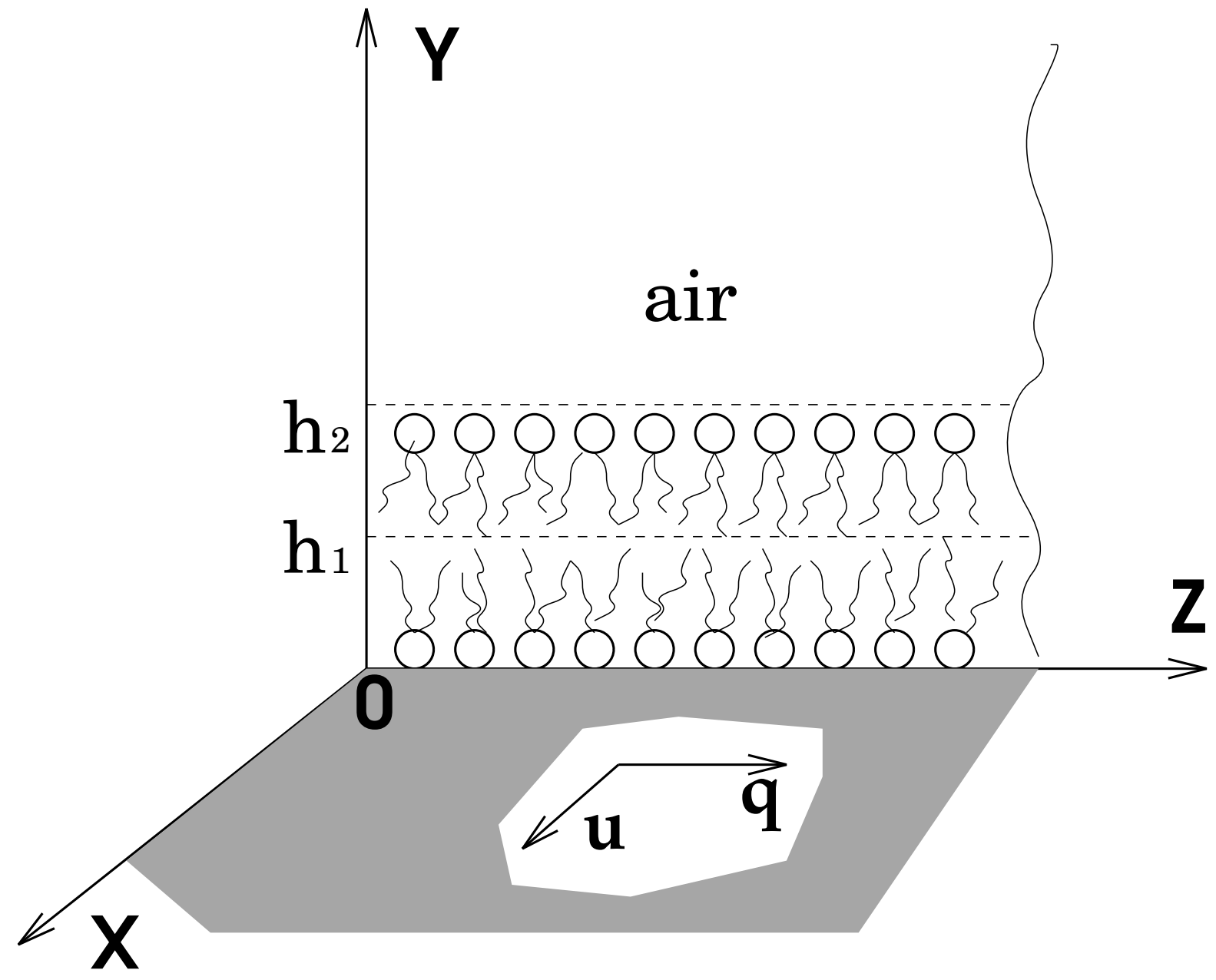

FIG. 1. Illustration of surface shear waves (SSW) with horizontal polarization propagating in a system of an elastic half-space with an adsorbed thin amphiphilic fluid bilayer on top. The system is in a gaseous enviroment. 


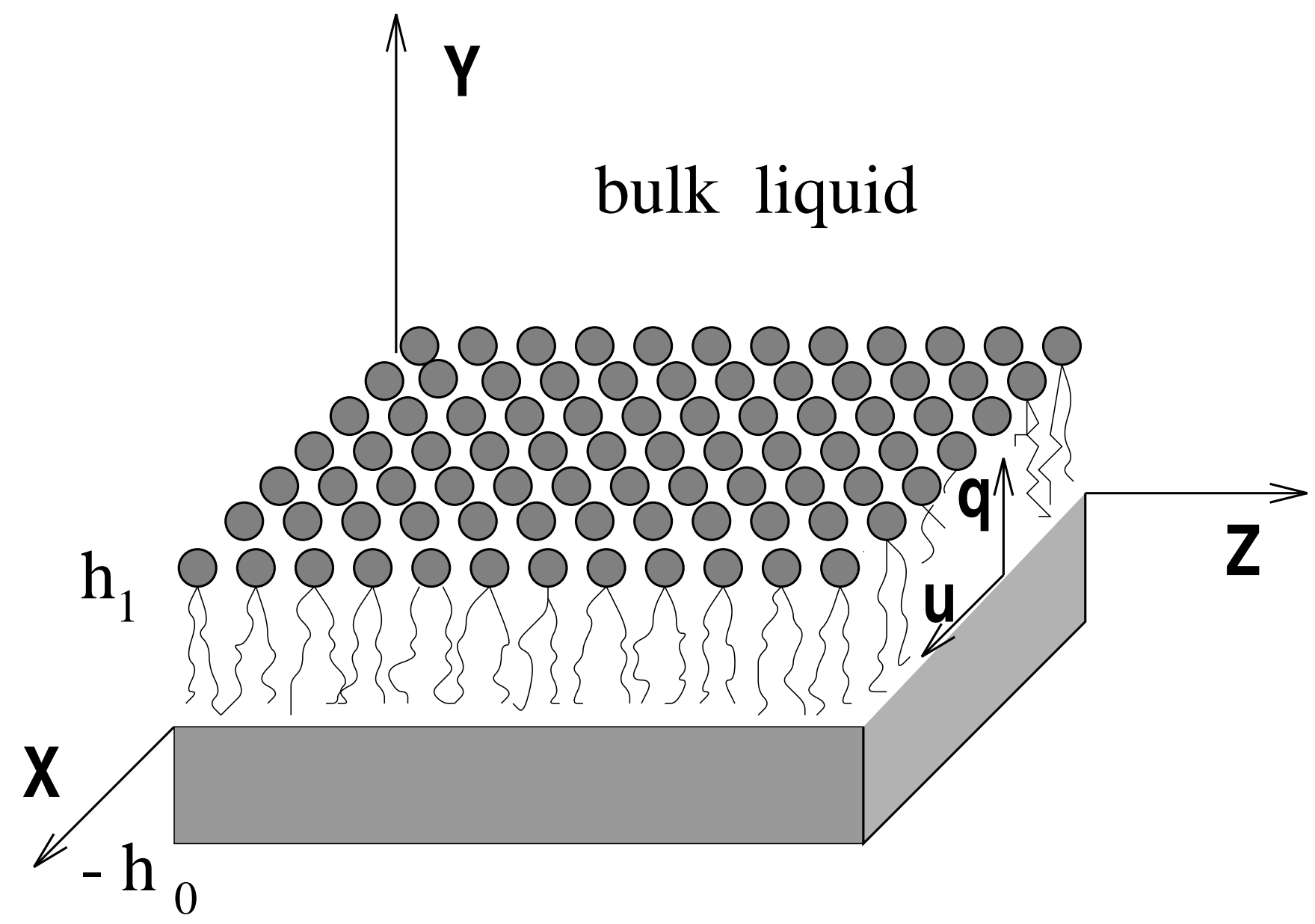

FIG. 2. shear bulk waves propagating in an AT-cut quartz plate covered with a thin amphiphilic layer. The system is immersed in a bulk liquid. 


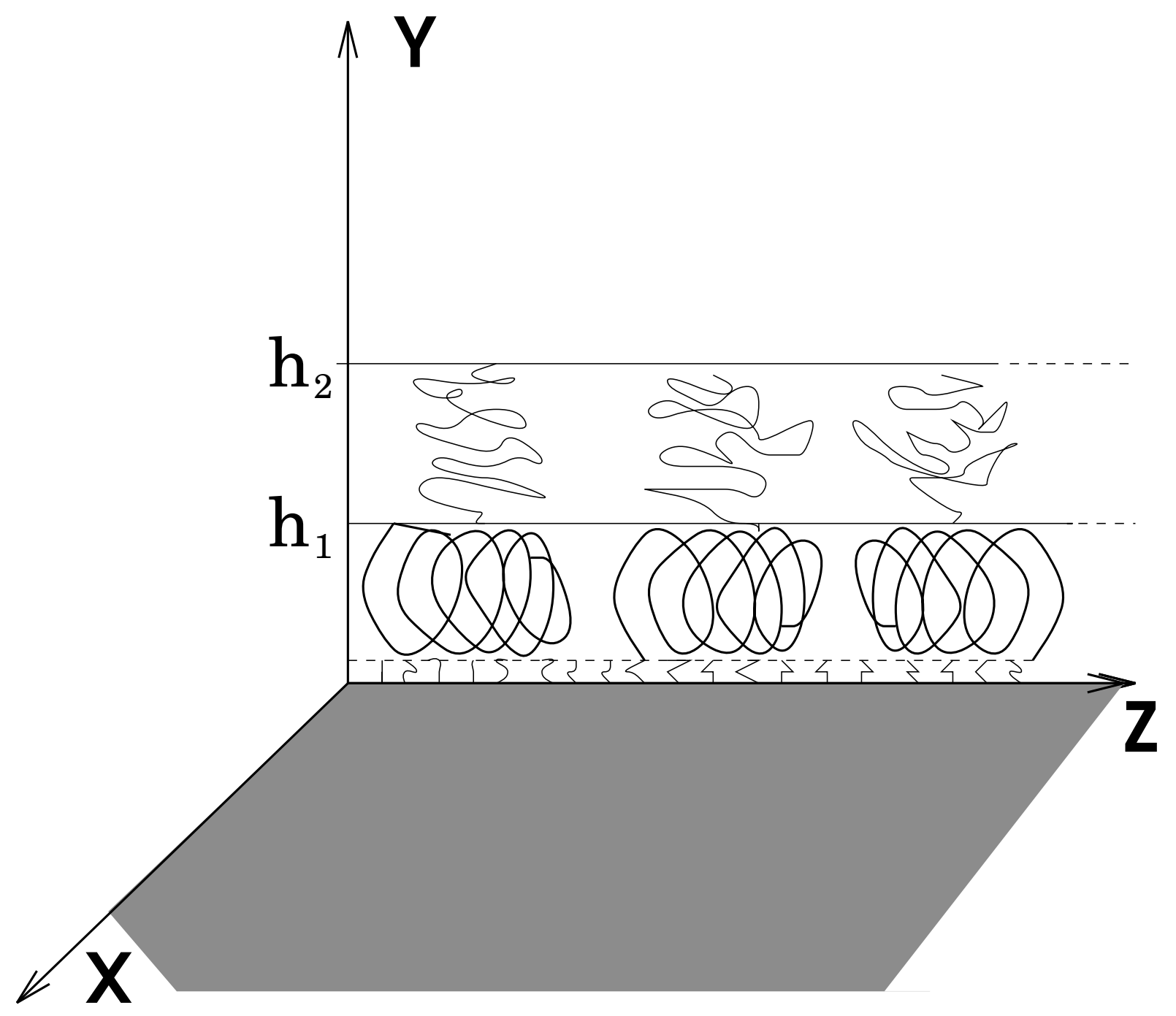

FIG. 3. Schematic depiction of two distinct regions of protein layer absorbed from bulk solution onto a solid substrate. The dotted line correspondes to the surface-adjacent amphiphilic layer. 